\title{
Document Classification for COVID-19 Literature
}

\author{
Bernal Jiménez Gutiérrez, Juncheng Zeng, Dongdong Zhang, Ping Zhang, Yu Su \\ The Ohio State University \\ \{jimenezgutierrez.1, zeng.671, zhang.11069, \\ zhang.10631, su.809\} dosu.edu
}

\begin{abstract}
The global pandemic has made it more important than ever to quickly and accurately retrieve relevant scientific literature for effective consumption by researchers in a wide range of fields. We provide an analysis of several multi-label document classification models on the LitCovid dataset, a growing collection of 23,000 research papers regarding the novel 2019 coronavirus. We find that pre-trained language models fine-tuned on this dataset outperform all other baselines and that BioBERT surpasses the others by a small margin with microF1 and accuracy scores of around $86 \%$ and $75 \%$ respectively on the test set. We evaluate the data efficiency and generalizability of these models as essential features of any system prepared to deal with an urgent situation like the current health crisis. We perform a data ablation study to determine how important article titles are for achieving reasonable performance on this dataset. Finally, we explore 50 errors made by the best performing models on LitCovid documents and find that they often (1) correlate certain labels too closely together and (2) fail to focus on discriminative sections of the articles; both of which are important issues to address in future work. Both data and code are available on GitHub ${ }^{1}$.
\end{abstract}

\section{Introduction}

The COVID-19 pandemic has made it a global priority for research on the subject to be developed at unprecedented rates. Researchers in a wide variety of fields, from clinicians to epidemiologists to policymakers, must all have effective access to the most up to date publications in their respective areas. Automated document classification can play an essential role in organizing the stream of articles by fields and topics to facilitate the search process and speed up research efforts.

\footnotetext{
${ }^{1}$ https://github.com/dki-lab/ covid19-classification
}

To explore how document classification models can help organize COVID-19 research papers, we use the LitCovid dataset (Chen et al., 2020), a collection of 23,000 newly released scientific papers compiled by the NIH to facilitate access to the literature on all aspects of the virus. This dataset is updated daily and every new article is manually assigned one or more of the following 8 categories: General, Transmission Dynamics (Transmission), Treatment, Case Report, Epidemic Forecasting (Forecasting), Prevention, Mechanism and Diagnosis. We leverage these annotations and the articles made available by LitCovid to compile a timely new dataset for multi-label document classification.

Apart from addressing the pressing needs of the pandemic, this dataset also offers an interesting document classification dataset which spans different biomedical specialties while sharing one overarching topic. This setting is distinct from other biomedical document classification datasets, which tend to exclusively distinguish between biomedical topics such as hallmarks of cancer (Baker et al., 2016), chemical exposure methods (Baker, 2017) or diagnosis codes (Du et al., 2019). The dataset's shared focus on the COVID-19 pandemic also sets it apart from open-domain datasets and academic paper classification datasets such as IMDB or the arXiv Academic Paper Dataset (AAPD) (Yang et al., 2018) in which no shared topic can be found in most of the documents, and it poses unique challenges for document classification models.

We evaluate a number of models on the LitCovid dataset and find that fine-tuning pre-trained language models yields higher performance than traditional machine learning approaches and neural models such as LSTMs (Adhikari et al., 2019b; Kim, 2014; Liu et al., 2017). We also notice that BioBERT (Lee et al., 2019), a BERT model pretrained on the original corpus for BERT plus a large set of PubMed articles, performed slightly better 


\begin{tabular}{lrr}
\hline & LitCovid & CORD-19 Test \\
\hline \# of Classes & 8 & 8 \\
\hline \# of Articles & 23,038 & 100 \\
\hline Avg. sentences & 74 & 109 \\
Avg. tokens & 1,399 & 2,861 \\
Total \# of tokens & $32,239,601$ & 286,065 \\
\hline
\end{tabular}

Table 1: Dataset statistics for the LitCovid and Test CORD-19 Datasets.

than the original BERT base model. We also observe that the novel Longformer (Beltagy et al., 2020) model, which allows for processing longer sequences, matches BioBERT's performance when 1024 subwords are used instead of 512, the maximum for BERT models.

We then explore the data efficiency and generalizability of these models as crucial aspects to address for document classification to become a useful tool against outbreaks like this one. Additionally, we perform a data ablation study to evaluate the effect of article titles on the limits of performance for this dataset. Finally, we discuss some issues found during our error analysis, such as current models often (1) correlating certain categories too closely with each other and (2) failing to focus on discriminative sections of a document and get distracted by introductory text about COVID-19, which suggest avenues for future improvement.

\section{Datasets}

In this section, we describe the LitCovid dataset in more detail and briefly introduce the CORD-19 dataset, which we sampled to create a small test set to evaluate model generalizability.

\subsection{LitCovid}

The LitCovid dataset is a collection of recently published PubMed articles that are directly related to the 2019 novel Coronavirus. The dataset contains upwards of 23,000 articles, and approximately 2,000 new articles are added every week, making it a comprehensive resource for keeping researchers up to date with the current COVID-19 crisis.

For a large portion of the articles in LitCovid, either the full article or at least the abstract can be downloaded directly from their website. For our document classification dataset, we select 23,038 articles which contain full texts or abstracts from the 35,000+ articles available on August $1^{\text {st }}, 2020$. As seen in table 1, these selected articles contain, on average, approximately 74 sentences and 1,399 tokens, reflecting the roughly even split between abstracts and full articles we observe from inspec-

\begin{tabular}{lrr}
\hline Class & LitCovid & CORD-19 Set \\
\hline Prevention & 11,042 & 12 \\
Treatment & 6,897 & 20 \\
Diagnosis & 4,754 & 25 \\
Mechanism & 3,549 & 70 \\
Case Report & 1,914 & 2 \\
Transmission & 1,065 & 6 \\
Forecasting & 461 & 2 \\
General & 368 & 7 \\
\hline
\end{tabular}

Table 2: Number of documents in each category for the LitCovid and CORD-19 Test Datasets.

tion.

Each article in LitCovid is assigned one or more of the following 8 topic labels: Prevention, Treatment, Diagnosis, Mechanism, Case Report, Transmission, Forecasting and General. Even though every article in the corpus can be labeled with multiple tags, most articles, around $76 \%$, contain only one label. Table 2 shows the label distribution for the subset of LitCovid, which is used in the present work. We note that there is a large class imbalance, with the most frequently occurring label appearing almost 20 times as much as the least frequent one. We split the LitCovid dataset into train, dev, test with the ratio $7: 1: 2$.

\subsection{CORD-19}

The COVID-19 Open Research Dataset (CORD19) (Wang et al., 2020) was one of the earliest datasets released to facilitate cooperation between the computing community and the many relevant actors of the COVID-19 pandemic. It consists of approximately 60,000 papers related to COVID19 and similar coronaviruses such as SARS and MERS since the SARS epidemic of 2002. Due to their differences in scope, this dataset shares only around 1,200 articles with the LitCovid dataset.

In order to test how our models generalize to a different setting, we asked biomedical experts to label a small set of 100 articles found only in CORD-19. Each article was labeled independently by two annotators. For articles that received two different annotations (around 15\%), a third annotator broke ties. Table 1 shows the statistics of this small set and Table 2 shows its category distribution.

\section{Models}

In the following section, we provide a brief description of each model and the implementations used. We use micro-F1 (F1) and accuracy (Acc.) as our evaluation metrics, as done in (Adhikari 


\begin{tabular}{lllll}
\hline \multirow{2}{*}{ Model } & \multicolumn{2}{c}{ Dev Set } & \multicolumn{2}{c}{ Test Set } \\
\cline { 2 - 5 } & Acc. & F1 & Acc. & F1 \\
\hline LR & 68.5 & 81.4 & 68.6 & 81.4 \\
SVM & 71.2 & 83.4 & 70.7 & 83.3 \\
\hline LSTM & $69.0 \pm 0.9$ & $83.9 \pm 0.1$ & $68.9 \pm 0.3$ & $83.2 \pm 0.2$ \\
LSTM $_{\text {Keg }}$ & $71.2 \pm 0.5$ & $83.9 \pm 0.3$ & $70.8 \pm 0.7$ & $83.6 \pm 0.5$ \\
KimCNN $_{\text {XML-CNN }}$ & $79.9 \pm 0.2$ & $83.3 \pm 0.3$ & $68.8 \pm 0.1$ & $82.7 \pm 0.1$ \\
\hline BERT $_{\text {base }}$ & $74.9 \pm 0.4$ & $84.1 \pm 0.2$ & $71.7 \pm 0.7$ & $83.5 \pm 0.3$ \\
BERT $_{\text {large }}$ & $75.1 \pm 3.9$ & $85.9 \pm 1.9$ & $74.4 \pm 2.7$ & $85.3 \pm 1.4$ \\
Longformer $_{\text {BioBERT + LSTM }}$ & $74.4 \pm 0.8$ & $85.6 \pm 0.5$ & $73.9 \pm 0.8$ & $85.5 \pm 0.5$ \\
BioBERT & $75.0 \pm 0.8$ & $85.2 \pm 0.3$ & $72.4 \pm 0.7$ & $85.0 \pm 0.4$ \\
\hline
\end{tabular}

Table 3: Performance for each model expressed as mean \pm standard deviation across three training runs.

et al., 2019a). All reproducibility information can be found in Appendix A.

\subsection{Traditional Machine Learning Models}

To compare with simpler but competitive traditional baselines, we use the default scikit-learn (Pedregosa et al., 2011) implementation of logistic regression and linear support vector machine (SVM) for multi-label classification, which trains one classifier per class using a one-vs-rest scheme. Both models use TF-IDF weighted bag-of-words as input.

\subsection{Conventional Neural Models}

Using Hedwig ${ }^{2}$, a document classification toolkit, we evaluate the following models: KimCNN (Kim, 2014), XML-CNN (Liu et al., 2017) as well as an unregularized and a regularized LSTM (Adhikari et al., 2019b). We notice that they all perform similarly and slightly better traditional methods.

\subsection{Pre-Trained Language Models}

Using the same Hedwig document classification toolkit, we evaluate the performance of DocBERT (Adhikari et al., 2019a) on this task with a few different pre-trained language models. We fine-tune BERT base, BERT large (Devlin et al., 2019) and BioBERT (Lee et al., 2019), a version of BERT base which was further pre-trained on a collection of PubMed articles. We find all BERT models achieve their best performance with their highest possible sequence length of 512 subwords. Additionally, we fine-tune the pre-trained Longformer (Beltagy et al., 2020) in the same way and find that it performs best when a maximum sequence length of 1024 is used.

As in the original Longformer paper, we use global attention on the [CLS] token for document

\footnotetext{
${ }^{2}$ https://github.com/castorini/hedwig
}

classification but find that performance improves by around $1 \%$ if we use the average of all tokens as input instead of only the [CLS] representation. We hypothesize that this effect can be observed because the LitCovid dataset contains longer documents on average that the Hyperpartisan dataset used in the original Longformer paper.

We find that all pre-trained language models outperform the previous traditional and neural methods by a sizable margin in both accuracy and microF1 score. The best performing model is BioBERT, which achieves a micro-F1 score of $86.2 \%$ and an accuracy of $75.2 \%$ on the test set.

Finally, we compare against an architecture proposed by (Mulyar et al., 2019) to process long documents in the clinical setting. This baseline splits full documents into segments of 512 subwords, encodes each of them separately using the ClinicalBERT model, and combines them using an LSTM module over each segment's [CLS] token. We replaced ClinicalBERT with BioBERT to more adequately fit our use case. We find that this baseline's F1 and accuracy scores are around 1\% and 3\% lower than the length limited BioBERT, respectively. This drop in performance indicates that the extra content in longer documents distracts this model from more salient information in the title and abstract of the article, which is more efficiently leveraged by the standalone BioBERT model.

\section{Results \& Discussion}

In this section, we explore data efficiency and model generalizability, present a data ablation study, and discuss potential ways to improve performance on this task in future work.

\subsection{Data Efficiency}

During a sudden healthcare crisis like this pandemic, it is essential for models to obtain useful results as soon as possible. Since labeling biomedical articles is a very time-consuming process, achieving peak performance using less data becomes highly desirable. We thus evaluate the data efficiency of these models by training each of the ones shown in Figure 1 using 1\%, 5\%, 10\%, 20\% and $50 \%$ of our training data and report the micro-F1 score on the dev set. When selecting the data subsets, we sample each category independently to make sure they are all represented.

We observe that pre-trained models are much more data-efficient than other models and that 


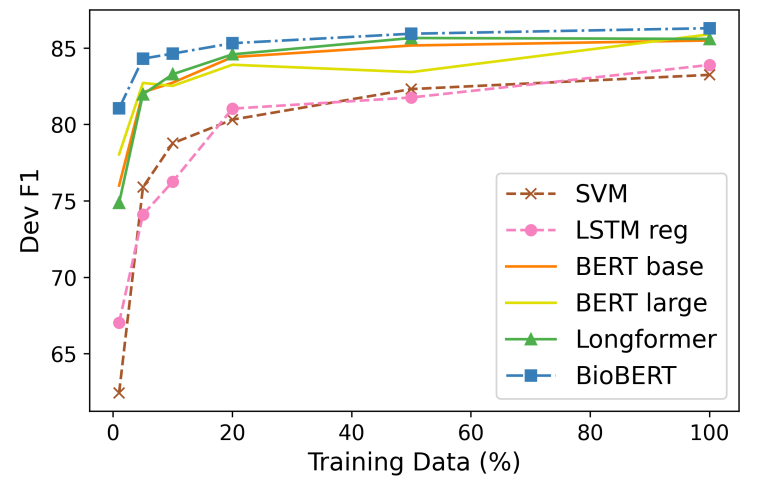

Figure 1: Data efficiency analysis. Pre-trained language models achieve their maximum performance on only $20 \%$ of the training data.

BioBERT is the most efficient, demonstrating the importance of domain-specific pre-training.

\subsection{CORD-19 Generalizability}

To effectively respond to this pandemic, experts must not only learn as much as possible about the current virus but also thoroughly understand past epidemics and similar viruses. Thus, it is crucial for models trained on the LitCovid dataset to successfully categorize articles about related epidemics. We therefore evaluate some of our baselines on such articles using our labeled CORD-19 subset. We find that the accuracy and micro-F1 metrics drop by approximately 35 and 15 points, respectively. This massive drop in performance from a minor change in domain indicates that the models have trouble ignoring the overarching COVID-19 topic and isolating relevant signals from each category.

\begin{tabular}{ccc}
\hline & Acc. & F1 \\
\hline SVM & 29.0 & 62.8 \\
LSTMreg & $32.7 \pm 1.5$ & $67.7 \pm 0.7$ \\
Longformer & $41.3 \pm 6.4$ & $70.0 \pm 2.9$ \\
BioBERT & $36.0 \pm 7.8$ & $69.7 \pm 2.8$ \\
\hline
\end{tabular}

Table 4: Performance on the CORD-19 Test Set expressed as mean \pm standard deviation across three training runs.

It is interesting to note that Mechanism is the only category for which BioBERT performs better in CORD-19 than in LitCovid. This could be due to Mechanism articles using technical language and there being enough samples for the models to learn; in contrast with Forecasting, which also uses specific language but has far fewer training examples. BioBERT's binary F1 scores for each category on both datasets can be found in Appendix B.

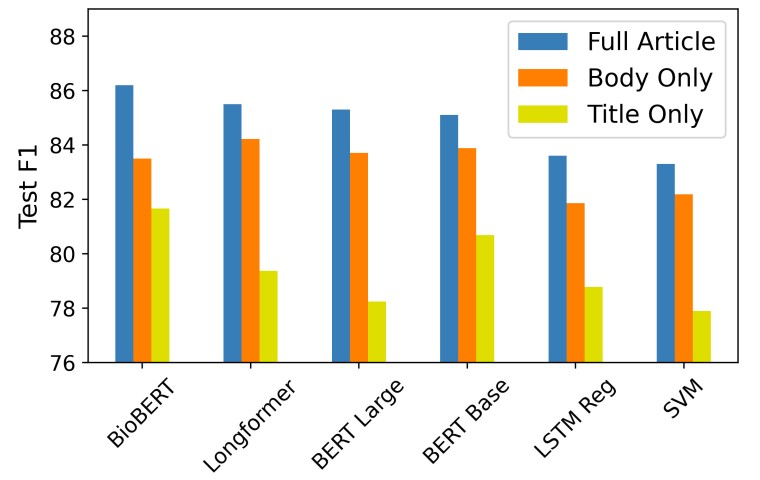

Figure 2: Data ablation study. Reasonable performance can be obtained with titles only but full articles are crucial for achieving best performance.

\subsection{Data Ablation Study}

To study whether full articles are required to achieve reasonable performance on this dataset, we evaluate a few representative models on two versions of the dataset, one with titles only and another with article bodies only. As we can see in Figure 2, the Longformer achieves the highest performance when titles are removed, given that it is able to process a larger portion of the article body than other pre-trained models. We also notice that the gap between BioBERT's 'Title Only' and 'Body Only' performance is much smaller than other models, suggesting that BioBERT's domain-specific pretraining allows it to use the salient title information more efficiently. We conclude that even though the article's body alone is more predictive than the title, standalone titles can still obtain reasonable performance on this dataset and are necessary to achieve the best possible performance.

\subsection{Error Analysis}

We analyze 50 errors made by both highest scoring BioBERT and the Longformer models on LitCovid documents to better understand their performance. We find that $34 \%$ of these were annotation errors which our best performing model predicted correctly. We also find that $10 \%$ of the errors were nearly impossible to classify using only the text available on LitCovid, and the full articles are needed to make better-informed prediction. From the rest of the errors we identify some aspects of this task which should be addressed in future work. We first note these models often correlate certain categories, namely Prevention and Forecasting, much more closely than necessary. Even though these categories are semantically related 


\begin{tabular}{|c|c|c|}
\hline Article & Label & Prediction \\
\hline $\begin{array}{l}\text { Analysis on epidemic situation and spatiotemporal changes of COVID-19 in Anhui. } \\
\text {... We mapped the spatiotemporal changes of confirmed cases, fitted the epidemic situation by the population growth } \\
\text { curve at different stages and took statistical description and analysis of the epidemic situation in Anhui province. }\end{array}$ & Forecasting & $\begin{array}{l}\text { Prevention } \\
\text { Forecasting }\end{array}$ \\
\hline $\begin{array}{l}2019 \text { Novel coronavirus: where we are and what we know. } \\
\text { There is a current worldwide outbreak of a new type of coronavirus (2019-nCoV), which originated from Wuhan in } \\
\text { China and has now spread to } 17 \text { other countries. } \\
\ldots \text { This paper aggregates and consolidates the virology, epidemiology, clinical management strategies ... } \\
\text { In addition, by fitting the number of infections with a single-term exponential model ... }\end{array}$ & $\begin{array}{l}\text { Treatment } \\
\text { Mechanism } \\
\text { Transmission } \\
\text { Forecasting }\end{array}$ & $\begin{array}{l}\text { Prevention } \\
\text { Forecasting }\end{array}$ \\
\hline $\begin{array}{l}\text { Managing Cancer Care During the COVID-19 Pandemic: Agility and Collaboration Toward a Common Goal. } \\
\text { The first confirmed case of coronavirus disease } 2019 \text { (COVID-19) in the United States was reported on } \\
\text { January 20, 2020, in Snohomish County, Washington. ... }\end{array}$ & Treatment & Prevention \\
\hline
\end{tabular}

Table 5: LitCovid Error Samples. Sentences relevant to the article's category are highlighted with blue and general ones with red.

and some overlap exists, the Forecasting tag is predicted in conjunction with the Prevention tag much more frequently than what is observed in the labels, as can be seen from the table in Appendix C. Future work should attempt to explicitly model correlation between categories to help the model recognize the particular cases in which labels should occur together. The first row in Table 5 shows a document labelled as Forecasting which is also incorrectly predicted with a Prevention label, exemplifying this issue.

Finally, we observe that models have trouble identifying discriminative sections of the document due to how much introductory content on the pandemic can be found in most articles. Future work should explicitly model the gap in relevance between introductory sections and crucial sentences such as thesis statements and article titles. In Table 5 , the second and third examples would be more easily classified correctly if specific sentences were ignored while others attended to more thoroughly. This could also increase interpretability, facilitating analysis and further improvement.

\section{Conclusion}

We provide an analysis of document classification models on the LitCovid dataset for the COVID19 literature. We determine that fine-tuning pretrained language models yields the best performance on this task. We study the generalizability and data efficiency of these models, evaluate the effect of article titles on performance through a data ablation study and discuss some important issues to address in future work.

\section{Acknowledgments}

This research was sponsored in part by the Ohio Supercomputer Center (Center, 1987). The authors would also like to thank Lang Li and Tanya BergerWolf for helpful discussions.

\section{References}

Ashutosh Adhikari, Achyudh Ram, Raphael Tang, and Jimmy Lin. 2019a. Docbert: Bert for document classification. ArXiv, abs/1904.08398.

Ashutosh Adhikari, Achyudh Ram, Raphael Tang, and Jimmy Lin. 2019b. Rethinking complex neural network architectures for document classification. In NAACL-HLT.

Simon Baker. 2017. Corpus and Software.

Simon Baker, Ilona Silins, Yufan Guo, Imran Ali, Johan Högberg, Ulla Stenius, and Anna Korhonen. 2016. Automatic semantic classification of scientific literature according to the hallmarks of cancer. Bioinformatics, 32 3:432-40.

Iz Beltagy, Matthew E. Peters, and Arman Cohan. 2020. Longformer: The long-document transformer. arXiv:2004.05150.

Ohio Supercomputer Center. 1987. Ohio supercomputer center.

Q. Chen, A. Allot, and Z. Lu. 2020. Keep up with the latest coronavirus research. Nature, 579(7798):193.

Jacob Devlin, Ming-Wei Chang, Kenton Lee, and Kristina Toutanova. 2019. Bert: Pre-training of deep bidirectional transformers for language understanding. ArXiv, abs/1810.04805.

Jingcheng Du, Qingyu Chen, Yifan Peng, Yang Xiang, Cui Tao, and Zhiyong Lu. 2019. Ml-net: multi-label classification of biomedical texts with deep neural networks. Journal of the American Medical Informatics Association : JAMIA.

Yoon Kim. 2014. Convolutional neural networks for sentence classification. In EMNLP.

Jinhyuk Lee, Wonjin Yoon, Sungdong Kim, Donghyeon Kim, Sunkyu Kim, Chan Ho So, and Jaewoo Kang. 2019. BioBERT: a pre-trained biomedical language representation model for biomedical text mining. Bioinformatics.

Jingzhou Liu, Wei-Cheng Chang, Yuexin Wu, and Yiming Yang. 2017. Deep learning for extreme multilabel text classification. Proceedings of the 40th 
International ACM SIGIR Conference on Research and Development in Information Retrieval.

Andriy Mulyar, Elliot Schumacher, M. Rouhizadeh, and Mark Dredze. 2019. Phenotyping of clinical notes with improved document classification models using contextualized neural language models. ArXiv, abs/1910.13664.

Fabian Pedregosa, Gaël Varoquaux, Alexandre Gramfort, Vincent Michel, Bertrand Thirion, Olivier Grisel, Mathieu Blondel, Gilles Louppe, Peter Prettenhofer, Ron Weiss, Vincent Dubourg, Jacob VanderPlas, Alexandre Passos, David Cournapeau, Matthieu Brucher, Matthieu Perrot, and Edouard Duchesnay. 2011. Scikit-learn: Machine learning in python. J. Mach. Learn. Res., 12:2825-2830.

Lucy Lu Wang, Kyle Lo, Yoganand Chandrasekhar, Russell Reas, Jiangjiang Yang, Darrin Eide, Kathryn Funk, Rodney Michael Kinney, Ziyang Liu, William. Merrill, Paul Mooney, Dewey A. Murdick, Devvret Rishi, Jerry Sheehan, Zhihong Shen, Brandon Stilson, Alex D. Wade, Kuansan Wang, Christopher Wilhelm, Boya Xie, Douglas M. Raymond, Daniel S Weld, Oren Etzioni, and Sebastian Kohlmeier. 2020. Cord-19: The covid-19 open research dataset. ArXiv, abs/2004.10706.

Pengcheng Yang, Xu Sun, Wei Li, Shuming Ma, Wei Wu, and Houfeng Wang. 2018. Sgm: Sequence generation model for multi-label classification. In $C O L$ ING. 


\section{A Experimental Set-up}

We split the LitCovid dataset into train, dev, test with the ratio $7: 1: 2$.

Following (Adhikari et al., 2019a), we adopt micro-F1 and accuracy as our evaluation metrics. We use scikit-learn (Pedregosa et al., 2011) and Hedwig evaluation scripts to evaluate all the models. We use the NLTK library for preprocessing, tokenization and sentence segmentation.

All the document classification models used in the paper, logistic regression ${ }^{1} \mathrm{SVM}^{2}$ DocBERT ${ }^{3}$, Reg-LSTM $^{4}$, Reg-LSTM $^{5}$, XML-CNN $^{6}$, Kim $\mathrm{CNN}^{7}$ are run based on the implementations listed here and strictly followed their instructions. We used the following pre-trained language models, BioBERT $^{8}$, BERT base ${ }^{9}$, BERT large ${ }^{10}$ and the Longformer ${ }^{11}$.

For reproducibility, we list all the key hyperparameters, the tuning bounds and the \# of parameters for each model in Table A3. For the logistic regression and the SVM all hyperparameters used were default to scikit-learn and therefore are excluded from this table. For all models, we train for a maximum of 30 epochs with a patience of 5 . We used the micro-F1 score for all hyperparameter tuning. All models were run on NVIDIA GeForce GTX 1080 GPUs.

\section{B Performance by Category}

In this section, we present BioBERT's binary F1 scores per category on both the LitCovid dev set and the CORD-19 test dataset. On the LitCovid dataset, BioBERT obtains scores above $80 \%$ for all

\footnotetext{
${ }^{1}$ https://scikit-learn.org/stable/ modules/generated/\sklearn. linear_model. LogisticRegression.html

${ }^{2}$ https://scikit-learn.org/stable/ modules/generated/sklearn.svm.SVC.html

${ }^{3}$ https://github.com/castorini/hedwig/ $\mathrm{blob} / \mathrm{master} / \mathrm{models} / \mathrm{bert}$

${ }^{4}$ https: / / github.com/castorini/hedwig/ blob/master/models/reg_lstm

${ }^{5}$ https: / / github. com/castorini/hedwig/ blob/master/models/reg_lstm

${ }^{6}$ https: / / github.com/castorini/hedwig/

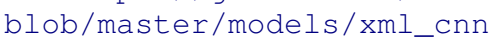

${ }^{7}$ https://github.com/castorini/hedwig/ $\mathrm{blob/master/models/kim \_ cnn}$

${ }^{8}$ https: / / huggingface.co/monologg/ biobert v1.1_pubmed

${ }^{9}$ https: // huggingface.co/ bert-base-uncased

${ }^{10}$ https: / / huggingface.co/ bert-large-uncased

${ }^{11}$ https://github.com/allenai/longformer
}

categories except Transmission, Forecasting, and General, the three categories with the least amount of training data. The class with the least number of training samples, General, has the lowest performance by far, obtaining half the F1 score than the second smallest class, Forecasting; this suggests that the General class is especially challenging. We note that Mechanism is the only category for which BioBERT does better in CORD-19 than in LitCovid. This is most likely due to the technical language used in the Mechanism category and the fact that it has more training examples than other highly technical categories such as Forecasting.

\begin{tabular}{ccc}
\hline Category & \multicolumn{2}{c}{ Binary F1 Score } \\
\cline { 2 - 3 } & $\begin{array}{c}\text { LitCovid } \\
\text { Dev }\end{array}$ & CORD-19 Set \\
\hline Prevention & $92.7 \pm 0.7$ & $66.7 \pm 2.5$ \\
Case Report & $87.9 \pm 1.5$ & $66.7 \pm 0.0$ \\
Treatment & $85.6 \pm 0.9$ & $53.9 \pm 9.0$ \\
Diagnosis & $82.1 \pm 0.5$ & $63.0 \pm 3.3$ \\
Mechanism & $81.5 \pm 2.1$ & $86.8 \pm 0.8$ \\
Forecasting & $70.3 \pm 2.3$ & $0.0 \pm 0.0$ \\
General & $35.5 \pm 33.9$ & $0.0 \pm 0.0$ \\
Transmission & $60.9 \pm 1.6$ & $56.4 \pm 3.2$ \\
\hline
\end{tabular}

Table A1: BioBERT Binary F1 scores per category on the LitCovid dev set and the CORD-19 test set. Scores are given as mean \pm standard deviation across three BioBERT training runs.

\section{Category Correlation}

Table A2 suggests that the Longformer model tends to predict the Prevention class whenever it predicts the Forecasting class. Although these categories are semantically similar and occur together in twothirds of the total Forecasting documents, they are predicted jointly in almost $90 \%$ of the documents labeled with the Forecasting class. This shows that these two classes are coupled more closely than they should; future work could explicitly address this coupling to improve performance.

\begin{tabular}{cccc}
\hline \multirow{2}{*}{ Category } & \multirow{2}{*}{ Full Label } & \multicolumn{2}{c}{$\begin{array}{c}\text { Percentage of Docs } \\
\text { with Category }\end{array}$} \\
\cline { 3 - 4 } & & Label & Prediction \\
\hline \multirow{2}{*}{ Forecasting } & Single Label & 34.9 & 8.5 \\
\cline { 2 - 4 } & + Prevention & 62.7 & 88.0 \\
\hline
\end{tabular}

Table A2: The numbers presented are percentages of the total number of documents with that category label. 


\begin{tabular}{|c|c|c|c|}
\hline Model & Hyperparameters & Hyperparameter bounds & Number of Parameters \\
\hline Kim CNN & $\begin{array}{l}\text { batch size: } 32 \\
\text { learning rate: } 0.001 \\
\text { dropout: } 0.1 \\
\text { mode: static } \\
\text { output channel: } 100 \\
\text { word dimension: } 300 \\
\text { embedding dimension: } 300 \\
\text { epoch decay: } 15 \\
\text { weight decay: } 0\end{array}$ & $\begin{array}{l}\text { batch size: }(16,32,64) \\
\text { learning rate: }(0.01,0.001,0.0001) \\
\text { dropout: }(0.1,0.5,0.7)\end{array}$ & 362,708 \\
\hline XML-CNN & $\begin{array}{l}\text { batch size: } 32 \\
\text { learning rate: } 0.001 \\
\text { dropout: } 0.7 \\
\text { dynamic pool length: } 8 \\
\text { mode: static } \\
\text { output channel: } 100 \\
\text { word dimension: } 300 \\
\text { embedding dimension: } 300 \\
\text { epoch decay: } 15 \\
\text { weight decay: } 0 \\
\text { hidden bottleneck dimension: } 512\end{array}$ & $\begin{array}{l}\text { batch size: }(32,64) \\
\text { learning rate: }\left(0.001,0.0001,1 \times 10^{-5}\right) \\
\text { dropout: }(0.1,0.5,0.7) \\
\text { dynamic pool length: }(8,16,32)\end{array}$ & $1,653,716$ \\
\hline LSTM & $\begin{array}{l}\text { batch size: } 8 \\
\text { learning rate: } 0.001 \\
\text { dropout: } 0.1 \\
\text { hidden dimension: } 512 \\
\text { mode: static } \\
\text { output channel: } 100 \\
\text { word dimension: } 300 \\
\text { embedding dimension: } 300 \\
\text { number of layers: } 1 \\
\text { epoch decay: } 15 \\
\text { weight decay: } 0 \\
\text { bidirectional: true } \\
\text { bottleneck layer: true } \\
\text { weight drop: } 0.1 \\
\text { embedding dropout: } 0.2 \\
\text { temporal averaging: } 0.0 \\
\text { temporal activation regularization: } 0.0 \\
\text { activation regularization: } 0.0\end{array}$ & $\begin{array}{l}\text { learning rate: }(0.01,0.001,0.0001) \\
\text { hidden dimension: }(300,512)\end{array}$ & $3,342,344$ \\
\hline LSTM $_{\text {Reg }}$ & $\begin{array}{l}\text { batch size: } 8 \\
\text { learning rate: } 0.001 \\
\text { dropout: } 0.5 \\
\text { hidden dimension: } 300 \\
\text { temporal averaging: } 0.99 \\
\text { mode: static } \\
\text { output channel: } 100 \\
\text { word dimension: } 300 \\
\text { embedding dimension: } 300 \\
\text { number of layers: } 1 \\
\text { epoch decay: } 15 \\
\text { weight decay: } 0 \\
\text { bidirectional: true } \\
\text { bottleneck layer: true } \\
\text { weight drop: } 0.1 \\
\text { embedding dropout: } 0.2 \\
\text { temporal activation regularization: } 0.0 \\
\text { activation regularization: } 0.0\end{array}$ & $\begin{array}{l}\text { batch size: }(8,16) \\
\text { learning rate: }(0.01,0.001,0.0001) \\
\text { hidden dimension: }(300,512) \\
\text { dropout: }(0.5,0.6)\end{array}$ & $1,449,608$ \\
\hline BERT $_{\text {base }}$ & $\begin{array}{l}\text { learning rate: } 2 \times 10^{-5} \\
\text { max sequence length: } 512 \\
\text { batch size: } 6 \\
\text { model: bert-base-uncased } \\
\text { warmup proportion: } 0.1 \\
\text { gradient accumulation steps: } 1\end{array}$ & $\begin{array}{l}\text { learning rate: }(0.001,0.0001 \text {, } \\
\left.2 \times 10^{-5}, 1 \times 10^{-6}\right) \\
\text { maximum sequence length: }(256,512)\end{array}$ & $110 \mathrm{M}$ \\
\hline BERT $_{\text {large }}$ & $\begin{array}{l}\text { learning rate: } 2 \times 10^{-5} \\
\text { max sequence length: } 512 \\
\text { batch size: } 2 \\
\text { model: bert-large-uncased } \\
\text { warmup proportion: } 0.1 \\
\text { gradient accumulation steps: } 1\end{array}$ & $\begin{array}{l}\text { learning rate: }(0.001,0.0001 \text {, } \\
\left.2 \times 10^{-5}, 1 \times 10^{-6}\right) \\
\text { maximum sequence length: }(256,512)\end{array}$ & $336 \mathrm{M}$ \\
\hline BioBERT & $\begin{array}{l}\text { learning rate: } 2 \times 10^{-5} \\
\text { max sequence length: } 512 \\
\text { batch size: } 6 \\
\text { model: monologg/biobert_v1.1_pubmed } \\
\text { warmup proportion: } 0.1 \\
\text { gradient accumulation steps: } 1\end{array}$ & $\begin{array}{l}\text { learning rate: }(0.001,0.0001 \text {, } \\
\left.\left.2 \times 10^{-5}, 1 \times 10^{-6}\right)\right) \\
\text { maximum sequence length: }(256,512)\end{array}$ & $108 \mathrm{M}$ \\
\hline BioBERT + LSTM & $\begin{array}{l}\text { learning rate: } 2 \times 10^{-5} \\
\text { max sequence length: } 512 \\
\text { batch size: } 16 \\
\text { model: monologg/biobert_v1.1_pubmed } \\
\text { weight decay: } 0 \\
\text { freeze bert: true }\end{array}$ & & $108 \mathrm{M}$ \\
\hline Longformer & $\begin{array}{l}\text { learning rate: } 2 \times 10^{-5} \\
\text { max sequence length: } 1024 \\
\text { batch size: } 3 \\
\text { model: longformer-base- } 4096 \\
\text { warmup proportion: } 0.1 \\
\text { gradient accumulation steps: } 1\end{array}$ & $\begin{array}{l}\text { learning rate: }(0.001,0.0001 \text {, } \\
\left.\left.2 \times 10^{-5}, 1 \times 10^{-6}\right)\right) \\
\text { maximum sequence length: }(1024,3584)\end{array}$ & $148 \mathrm{M}$ \\
\hline
\end{tabular}

Table A3: Hyperparameters, tuning bounds and number of parameters for each method. 